\title{
Primary Reinforcing Effects of Nicotine Are Triggered from Multiple Regions Both Inside and Outside the Ventral Tegmental Area
}

\author{
Satoshi Ikemoto, Mei Qin, and Zhong-Hua Liu \\ Behavioral Neuroscience Branch, National Institute on Drug Abuse, National Institutes of Health, Department of Health and Human Services, Baltimore, \\ Maryland 21224
}

\begin{abstract}
Nicotine is thought to be the key substance responsible for tobacco-smoking habits and appears to trigger reinforcement via the ventral tegmental area (VTA). Recently, multiple anatomical substrates for drug reinforcement have been identified in the vicinity of the ventral midbrain. In addition to the posterior portion of the VTA, the central linear nucleus raphe and the supramammillary nucleus of the posterior hypothalamus mediate drug reinforcement. Using intracranial self-administration procedures, we examined whether these regions mediate the reinforcing effects of nicotine. Rats learned to lever press for self-administration of nicotine into the posterior VTA, central linear nucleus, and supramammillary nucleus, suggesting a reinforcing action of nicotine in these regions. The rats did not self-administer nicotine into surrounding regions including the anterior VTA, substantia nigra, the region just dorsal to the posterior VTA, interpeduncular nucleus, or medial mammillary nucleus. The reinforcing effects of nicotine into the three brain regions were further confirmed by a two-lever discrimination procedure, in which rats learned to selectively respond between active and inactive levers. The reinforcing effects of nicotine administration into the posterior VTA, central linear nucleus, and supramammillary nucleus were blocked by coadministration of the nicotine receptor antagonist mecamylamine. The reinforcing effects of nicotine into the posterior VTA or central linear nucleus were attenuated by coadministration of the $\mathrm{D}_{2}$ receptor agonist quinpirole. These findings demonstrate that nicotine reinforcement involves multiple regions both inside and outside the VTA.
\end{abstract}

Key words: nicotinic cholinergic receptor; supramammillary nucleus; central linear nucleus raphé; caudal linear nucleus raphé; reward; intracranial self-administration

\section{Introduction}

Nicotine, found in tobacco leaves, is thought to be the key substance responsible for smoking habits. A series of studies in animals suggests that nicotine stimulates nicotinic cholinergic receptors to trigger its reinforcing effects, which involve dopaminergic systems (Di Chiara, 2000; Picciotto and Corrigall, 2002). A key brain region mediating nicotine reward appears to be the ventral tegmental area (VTA). Rats quickly learn to press levers for local infusions of the nonselective cholinergic agonist carbachol or the acetylcholinesterase inhibitor neostigmine into the VTA; these reinforcing effects are attenuated by coadministration of nicotinic receptor antagonists (Ikemoto and Wise, 2002). Indeed, a recent study showed that mice learn to run on a Y-maze for nicotine administration into the VTA (Maskos et al., 2005). Intravenous self-administration of nicotine in rats is attenuated by the blockade of nicotinic receptors in the VTA (Cor-

Received 0ct. 24, 2005; revised Nov. 19, 2005; accepted Nov. 20, 2005.

This work was supported by the Intramural Research Program of the National Institutes of Health, National Institute on Drug Abuse. We are grateful for the technical assistance of David Horton, Ian Macumber, and Robert Ator and for the editorial assistance of Emily Wentzell.

Correspondence should be addressed to Satoshi lkemoto, National Institute on Drug Abuse, Behavioral Neuroscience Branch, 5500 Nathan Shock Drive, Baltimore, MD 21224. E-mail: sikemoto@intra.nida.nih.gov.

DOI:10.1523/JNEUROSCI.4542-05.2006

Copyright $\odot 2006$ Society for Neuroscience $\quad$ 0270-6474/06/260723-08\$15.00/0 rigall et al., 1994) as well as by selective lesions of the VTA neurons projecting to the ventral striatum (Corrigall et al., 1992). In addition, administration of the nicotinic receptor agonists cytisine or nicotine into the vicinity of the VTA induces conditioned place preference (Museo and Wise, 1994; Laviolette and van der Kooy, 2003), which demonstrates its rewarding effects.

Recent studies suggest that the organization of the VTA and its adjacent regions in drug reinforcement is more complex than previously thought. First, the VTA appears to be functionally heterogeneous. The posterior portion of the VTA has been shown to be more responsible than the anterior VTA for the reinforcing effects of several drugs including opiates (Zangen et al., 2002), ethanol (Rodd-Henricks et al., 2000), and its metabolite acetaldehyde (Rodd et al., 2004). More importantly, the posterior VTA is more responsible for carbachol reward than the anterior VTA (Ikemoto and Wise, 2002). Studies using viral technology also indicate that the anterior and posterior VTA play different roles in modulating the rewarding properties of opiates and cocaine (Carlezon et al., 2000; Bolanos et al., 2003; Olson et al., 2005). These findings led us to hypothesize that rats would readily learn to self-administer nicotine into the posterior VTA but not the anterior VTA.

In addition, two regions localized adjacent to the VTA may also be important for reinforcing effects of nicotine. The central 
linear nucleus raphé, a midline structure just posterodorsal to the VTA, contains dopaminergic neurons projecting to the nucleus accumbens (Swanson, 1982). This site appears to play a role in positive reinforcement because rats learn to self-administer the $\mathrm{GABA}_{\mathrm{A}}$ receptor agonist muscimol into this region (Ikemoto et al., 1998b). Another possibly important region is the supramammillary nucleus, a midline structure in the posterior hypothalamic area just dorsal to the mammillary body and just anterior to the VTA. Rats learn to self-administer the excitatory amino acid AMPA (Ikemoto et al., 2004) or the $\mathrm{GABA}_{\mathrm{A}}$ receptor antagonists picrotoxin or bicuculline (Ikemoto, 2005a) into the supramammillary nucleus.

These findings suggest that a complex chemical reinforcement mechanism exists in the vicinity of the VTA. Precise action sites of nicotine for its primary reinforcement have not yet been investigated. To this end, we examined the VTA and adjacent regions using intracranial self-administration procedures in rats.

\section{Materials and Methods}

Subjects. One hundred and twenty-eight male Wistar rats (250-350 g at the time of surgery; Harlan, Dublin, VA) were initially housed in pairs in a colony room kept at a constant temperature $\left(21^{\circ} \mathrm{C}\right)$, on a reversed $12 \mathrm{~h}$ light/dark cycle (lights on at 9:30 P.M.). After surgery, the animals were individually housed. Food and water were available ad libitum to the animals except during testing. The procedures described in this study were approved by the Animal Care and Use Committee of the National Institute on Drug Abuse Intramural Research Program and were in accordance with National Institutes of Health guidelines.

Surgery. Under sodium pentobarbital (31 mg/kg, i.p.) and chloral hydrate $(142 \mathrm{mg} / \mathrm{kg}$, i.p.) anesthesia, each animal was implanted with a unilateral guide cannula (24 gauge) that ended $1.0 \mathrm{~mm}$ above one of target sites. Guide cannulas were inserted at a $6^{\circ}$ angle from the vertical toward the midline to avoid damaging the midline sinuses, except for the substantia nigra placement, in which guides were inserted at a $0^{\circ}$ angle. Posterior distance from bregma, lateral distance from the midline, and ventral distance from skull surface were measured along the trajectory of the angled cannula. These respective coordinates (in $\mathrm{mm}$ ) were: 5.4, 1.3, and 8.0 for posterior VTA; 5.7, 0.9, and 7.6 for central linear nucleus; $4.3-4.8,1.5-2.0$, and $7.8-8.0$ for anterior VTA; $4.0-4.5,1.0-1.3$, and 8.3 for supramammillary nucleus; $3.4-4.0,1.0-1.3$, and 7.6 for posterior hypothalamic nucleus; $4.2,1.4$, and 9.2 for medial mammillary nucleus; 5.6, 1.5-1.7, and 7.5-7.9 for substantia nigra; 5.4-5.7, 1.3, and 7.6 for dorsal to posterior VTA/lateral to central linear nucleus; and 6.0, 0.9, and 8.2 for interpeduncular nucleus. The incisor bar was set at $3.3 \mathrm{~mm}$ below the interaural line. Testing started 5-7 d after surgery.

Drugs. (-)Nicotine hydrogen tartate salt, mecamylamine hydrochloride, and ( - methyl scopolamine bromide (Sigma, St. Louis, MO) were dissolved in an artificial CSF consisting of the following (in mM): 148 $\mathrm{NaCl}, 2.7 \mathrm{KCl}, 1.2 \mathrm{CaCl}_{2}$, and $0.85 \mathrm{MgCl}_{2}, \mathrm{pH}$ adjusted to 7.4 with $\mathrm{NaOH}$ solutions.

Self-administration apparatus. Each operant chamber $(30 \times 22 \times 24$ $\mathrm{cm}^{3}$ ) was equipped with a lever $(45 \mathrm{~mm}$ wide $\times 2 \mathrm{~mm}$ thick; protruding $19 \mathrm{~mm}$ from the wall) just below a light assembly (25-mm-diameter disk) and was enclosed in an insulated box equipped with a ventilating fan. Each rat's 31 gauge injection cannula was connected by polyethylene tubing to a micropump (Ikemoto and Sharpe, 2001) hanging a few millimeters above the rat's head. Each pump consisted of a miniature step motor and a small plastic reservoir and was connected to an electrical swivel, which prevented the cable from being tangled and allowed the rats to move freely in the chamber. When activated, the motor advanced a shaft into the reservoir, displacing drug solution into the injection cannula.

Anatomical substrates of nicotine self-administration. A response on the lever in the operant chamber caused an intracranial injection ( $75 \mathrm{nl})$ over $5 \mathrm{~s}$ and turned on a tone, then extinguished the light above the lever and retracted the lever for $10 \mathrm{~s}$; these physical changes in the environment served to facilitate contingency learning between lever pressing and the effects of drug delivery. Each rat received drug infusions into one of eight regions: the supramammillary nucleus, medial mammillary nucleus, posterior hypothalamic nucleus, anterior VTA, posterior VTA, central linear nucleus, region dorsal to posterior VTA/lateral to central linear nucleus, and substantia nigra. Rats received vehicle in session 1 and were trained to lever press with injections of $25 \mathrm{~mm}$ nicotine in two sessions, followed by a vehicle session. The rates of self-administration (infusions per minute) during this acquisition phase were analyzed by an eight-bytwo-by-two mixed ANOVA with region (between-subjects factor), treatment, and trial (within-subjects factors). Then, we examined the effects of high nicotine concentrations of $12.5,25$, and $50 \mathrm{~mm}$ made available over three sessions in this order. We used an eight-by-three mixed ANOVA with region (as a between-subjects factor) and concentration (as a within-subjects factor) to analyze self-administration rates. After another vehicle session, we examined the effects of low nicotine concentrations of $0.1,0.3,1,3$ and $10 \mathrm{~mm}$ nicotine; each rat received this set of concentrations in this order over five sessions. We used an eight-by-five mixed ANOVA with region and concentration as factors to analyze selfadministration rates. Subsequently, we examined five additional rats using a procedure identical to that described above for the effects of nicotine administration into the interpeduncular nucleus, which was not included in the above statistical analysis. Sessions lasted for $90 \mathrm{~min}$ or until 60 infusions were earned. The number of infusions per session was limited to a total of 60 to minimize possible adverse physiological effects.

Two-lever discrimination experiment for nicotine reinforcement. Thirtyfive rats with no previous operant training were placed in operant conditioning chambers with two retractable levers. The chambers were identical to the one-lever chambers described above, except that two levers were placed on the chamber wall. A response on the "active" lever resulted in a $5 \mathrm{~s}$ infusion (volume, $75 \mathrm{nl}$ ), turned on a tone, extinguished the light above the lever, and retracted both levers for $20 \mathrm{~s}$. A response on the "inactive" lever did not deliver infusions but retracted both the active and inactive levers for $20 \mathrm{~s}$. The assignment of left and right levers for active and inactive functions was counterbalanced among subjects. Each rat received nicotine into one of the three sites: the posterior VTA, the central linear nucleus, and the supramammillary nucleus. The rats received vehicle in session 1 and $25 \mathrm{~mm}$ nicotine in sessions $2-4$. Sessions lasted 90 min or until the rats received a total of 60 infusions. The numbers of responses on each lever and subsequent infusions were recorded. A session (4)-by-lever (2) repeated ANOVA was conducted on lever presses for each region.

Pharmacological specificity. A group of experimentally naive rats was used to examine the roles of nicotine receptors and dopamine receptors in nicotine reward of the posterior VTA, with the one-lever procedure. Testing in this group was conducted slightly differently from others, because it was done before finding the reinforcing effects of nicotine in the central liner nucleus or supramammillary nucleus and before conducting the two-lever experiment described above. Using the rats we used in the two-lever discrimination experiment, we conducted the rest of testing described below.

We examined whether the reinforcing effects of nicotine are mediated via nicotinic receptors using the nicotinic receptor antagonist mecamylamine. We also examined the role of muscarinic cholinergic receptors in nicotine reward for the posterior VTA but not for the central linear or supramammillary nuclei. With the single-lever procedure described above, seven rats with no previous operant training were initially trained to self-administer $25 \mathrm{~mm}$ nicotine into the posterior VTA for three or four sessions and then received $25 \mathrm{~mm}$ nicotine, $25 \mathrm{~mm}$ nicotine plus $10 \mathrm{~mm}$ mecamylamine, $25 \mathrm{~mm}$ nicotine plus $1 \mathrm{~mm}$ scopolamine, and vehicle over four sessions. The order of testing these treatments was counterbalanced among the rats. A one-way repeated ANOVA with these four treatments was conducted on self-administration rates. For the supramammillary nucleus and central linear nucleus, the effects of mecamylamine were examined with the two-lever procedure described above. The rats that were used for the two-lever discrimination experiment received $25 \mathrm{~mm}$ nicotine, $25 \mathrm{~mm}$ nicotine plus $10 \mathrm{~mm}$ mecamylamine, and vehicle over three sessions. The order of testing these drugs was counterbalanced. One-way repeated ANOVAs with these three treatments were conducted on self-administration rates. 
To evaluate the effects of systemic administration of the dopamine receptor antagonist $R(+)$-7-chloro-8-hydroxy-3-methyl-1-phenyl2,3,4,5-tetrahydro- $1 \mathrm{H}$-3-benzazepine hydrochloride (SCH 23390), we used the seven posterior VTA rats that were used in the mecamylaminescopolamine experiment. Again, the single-lever procedure was used. In session 1 , the rats were pretreated with $0.9 \%$ saline $(1 \mathrm{ml} / \mathrm{kg}$, i.p. $)$ and received vehicle $30 \mathrm{~min}$ later. In sessions $2-5$, the rats were pretreated with SCH $23390(0.05 \mathrm{mg} / \mathrm{kg}$, i.p. $)$ or saline alternately and received 25 mM nicotine in sessions 2 and 3 and $0.3 \mathrm{~mm}$ nicotine in sessions 4 and 5 . A two-way repeated ANOVA with treatment (SCH 23390 vs saline) and nicotine concentration ( 0.3 vs $25 \mathrm{~mm})$ was conducted on selfadministration rates. In addition, to better understand the effects of SCH 23390 on self-administration of $25 \mathrm{~mm}$ nicotine and to determine rate differences between the beginning and end of sessions as a function of treatment conditions, we analyzed the first three infusion intervals and the last three infusion intervals as a function of treatments: nicotine infusion with SCH 23390, nicotine infusion with saline pretreatment, or vehicle infusion with saline pretreatment. Infusion interval data, however, were highly varied and, therefore, were square root transformed to help maintain homogeneity of variance for a three-way repeated ANOVA with interval (beginning vs end), trial (3), and treatment condition (3).

We evaluated the effects of selective inactivation of dopamine neurons using quinpirole in the rats used for the two-lever discrimination experiment for the posterior VTA and central linear nucleus. The rats were given the opportunities to self-administer $25 \mathrm{~mm}$ nicotine, $25 \mathrm{~mm}$ nicotine plus $0.1 \mathrm{~mm}$ quinpirole, and vehicle over three sessions with the two-lever procedure described above. The order of testing these treatments was counterbalanced. A one-way repeated ANOVA with treatment was conducted on self-administration rates.

Histology. When each rat completed the experimental procedure, it was deeply anesthetized with a mixture of sodium pentobarbital (31 $\mathrm{mg} / \mathrm{kg}$ ) and chloral hydrate $(142 \mathrm{mg} / \mathrm{kg})$, and its brain was removed and placed in a $4 \%$ paraformaldehyde solution. Coronal sections $(40 \mu \mathrm{m})$ at the microinjection site were cut with a cryostat. Sections were stained with cresyl violet. The placements of the injection cannulas were confirmed by microscopic examination.

Post hoc analyses. Unless other tests are mentioned, main effects that were found to be significant with ANOVAs were subjected to NewmanKeuls post hoc tests.

\section{Results}

\section{Anatomical substrates of nicotine self-administration}

To determine the precise anatomical substrates that mediate the primary reinforcing effects of nicotine, rats were trained to selfadminister nicotine into several different regions. Rats quickly learned to self-administer nicotine into the posterior VTA $(n=$ 13 ) and the central linear nucleus $(n=10)$ (Fig. $1 A)$. Within the VTA, the posterior VTA is more responsible for nicotine reinforcement than the anterior VTA $(n=21)$. As shown in Figure $1 \mathrm{~B}$, the portion of the posterior VTA just above the interpeduncular nucleus or the paranigral nucleus [as defined by Phillipson (1979)] and its immediate vicinity were most responsible for nicotine reward. When nicotine was delivered to more posterior sites than the paranigral nucleus at the level of $\sim 6.3 \mathrm{~mm}$ from bregma, nicotine was not reliably self-administered. In addition, nicotine was not self-administered into the substantia nigra $(n=$ 9) or the region just dorsal to the posterior VTA and just lateral to the central linear nucleus $(n=9)$. We also examined additional rats $(n=5)$ to determine whether the interpeduncular nucleus is responsible for reinforcing effects of nicotine and found that it was not (Fig. $1 B$ ). Interpeduncular rats did not self-administer any concentration of nicotine at rates higher than 0.16 . Therefore, the zones responsible for nicotine reward in the ventral midbrain appear to be confined to the posteromedial areas centered around the paranigral nucleus and the central linear nucleus.
In addition, supramammillary rats learned to respond for nicotine $(n=9)$. The findings that rats did not readily selfadminister nicotine into neighboring regions including the medial mammillary nucleus $(n=6)$, the posterior hypothalamic nucleus $(n=8)$, or the anterior VTA suggest that the reinforcing effects of nicotine in the posterior hypothalamic area is confined to the area centered around the supramammillary nucleus. These observations are confirmed by statistical analyses (Fig. 1, legend). It should be noted that the supramammillary nucleus may be less sensitive to the rewarding effects of nicotine than the posterior VTA or central linear nucleus, because supramammillary rats appeared to be less responsible for nicotine during the acquisition phase and for the low concentrations of nicotine in session 9-13 than the posterior VTA or central linear rats.

\section{Two-lever discrimination}

To determine the validity of the alternative interpretation that nicotine injections into the posterior VTA, central linear nucleus, or the supramammillary nucleus are merely arousing rather than reinforcing, we examined whether rats learn to discriminate between active and inactive levers for nicotine reward for each of these three effective regions. As shown in Figure 2, when rats received vehicle in the first session, they did not show significant preference for one lever over the other. When they received nicotine (25 mM) in sessions 2-5, they learned to respond on the active lever more than on the inactive lever. Response levels on the inactive lever stayed the same throughout sessions 1-5, whereas response levels on the active lever selectively increased over the sessions. Similar responding patterns over the sessions were observed across the three regions. These observations are confirmed with statistical analyses (Fig. 2, legend).

It should be noted that the self-administration rates demonstrated by these rats are considerably lower than those of the rats tested with the one-lever procedure in the previous experiment. For example, in the one-lever procedure, the rats selfadministered $25 \mathrm{~mm}$ nicotine in session 6 at the rates of $0.56,0.53$, and 0.35 infusions per minute into the posterior VTA, central linear nucleus, and supramammillary nucleus, respectively, whereas in the two-lever procedure, the rats self-administered it in session 5 at $0.27,0.31$, and 0.24 , respectively. This issue is addressed in the Discussion.

\section{Nicotinic receptor mediation}

To determine whether nicotine self-administration was mediated via nicotinic receptors, we examined the effect of nicotinic receptor antagonist mecamylamine injection into the three regions. In addition, we examined the effects of injection of the muscarinic cholinergic receptor antagonist scopolamine into the posterior VTA, because we found in our previous study that the blockade of muscarinic receptors attenuates reinforcing effects of the nonselective cholinergic agonist carbachol in the VTA (Ikemoto and Wise, 2002), and examined whether coactivation of muscarinic receptors is important for reinforcement. The reinforcing effects of nicotine injections into the posterior VTA were attenuated by coadministration of mecamylamine (Fig. 3), suggesting that nicotinic receptors mediate nicotine reinforcement. Coadministration of scopolamine also tended to attenuate the reinforcing effects of nicotine, although the differences were not statistically significant. Similarly, self-administration was attenuated when nicotine administration into the central linear nucleus or supramammillary nucleus was substituted with administration of a mix- 

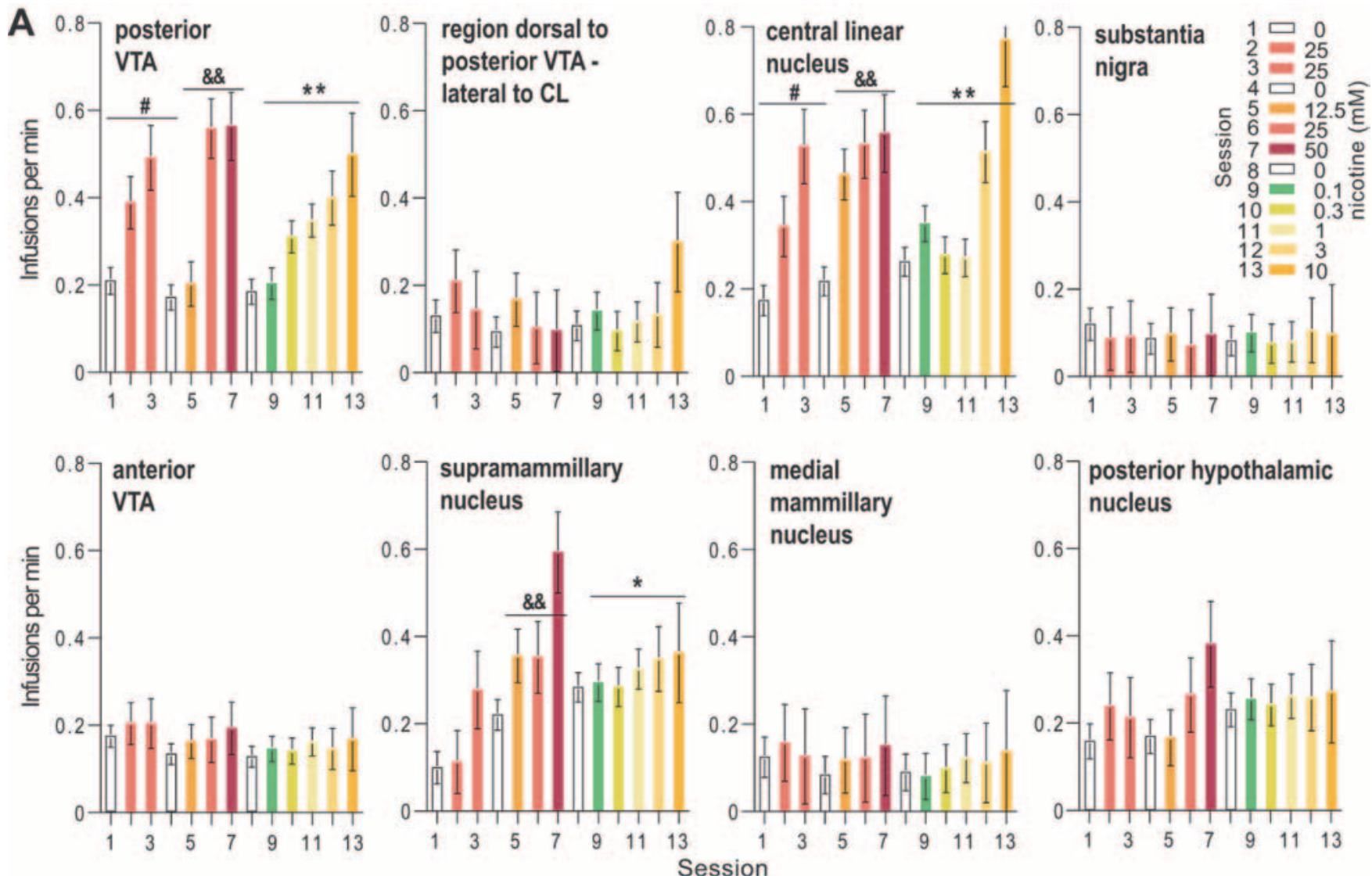

(Figure 1 continues).

ture of nicotine and mecamylamine (Fig. 3), suggesting that nicotinic receptors mediate nicotine reinforcement in these regions as well.

\section{Role of dopamine in midbrain nicotine reward}

Dopamine transmission has been implicated in the reinforcing effects of a number of drugs including amphetamines, cocaine, and nicotine. However, a recent study reported that the blockade of dopamine transmission can enhance the rewarding effects of nicotine, particularly at low doses, administered into the VTA (Laviolette and van der Kooy, 2003). Therefore, we examined the role of dopamine in the reinforcing effects of low and high concentrations of nicotine administered into the posterior VTA, using the dopamine receptor antagonist SCH 23390.

Pretreatment with SCH 23390 (0.05 mg/kg, i.p.) caused cessation of self-administration of the high nicotine concentration (Fig. 4A). The rats started out normally self-administering $25 \mathrm{~mm}$ nicotine, but a few minutes into the self-administration session, they decreased the rates of self-administration and responded less and less frequently (Fig. 4B,C). This self-administration pattern resembled that which occurred when the rats received vehicle substituted for nicotine; the pattern is thus extinction-like. Our observations were confirmed with a statistical analysis on the first three intervals of infusions and the last three infusion intervals that were compared for three different treatments, nicotine infusion with the $\mathrm{D}_{1}$ antagonist pretreatment, nicotine infusion with saline pretreatment, or vehicle infusion with saline pretreatment (Fig. 4B). The results of this analysis suggest that animals under the dopamine antagonist treatment were capable of responding, as indicated by normal rates of self-administration during the first several infusions. Therefore, the reduced self-administration rates toward the end of sessions were most likely caused by motivational deficit rather than motor deficit. Similarly, when rats received the low nicotine concentration $(0.3 \mathrm{~mm})$, which was insufficiently reinforcing to support persistent selfadministration throughout the session, the pretreatment with SCH 23390 (0.05 mg/kg, i.p.) tended to further reduce selfadministration rates (Fig. $4 A, C$ ). Thus, systemic blockade of dopamine receptors appears to block the reinforcing effects of intraVTA nicotine, at both high and low concentrations.

To determine whether the reinforcing effects of nicotine are mediated via local dopamine neurons at the injection sites, we administered the dopamine $\mathrm{D}_{2}$ receptor agonist quinpirole together with nicotine. When a mixture of nicotine plus quinpirole was substituted for nicotine alone, self-administration into the posterior VTA or central linear nucleus was significantly attenuated (Fig. 4D). This suggests that local dopaminergic neurons play a critical role in mediating nicotine reinforcement in the posterior VTA and central linear nucleus.

\section{Discussion}

We found that the reinforcing effects of nicotine are triggered via multiple brain regions in the vicinity of the ventromedial midbrain. There appear to be pockets of areas implicated in reinforcement surrounded by uninvolved areas. One of the sites most responsible for nicotine reinforcement is the posterior portion of the VTA. Rats quickly learned to self-administer nicotine into the posterior VTA, and a wide range of nicotine concentrations were self-administered into this site. Similarly, nicotine was reliably self-administered into the central linear nucleus raphé and the 

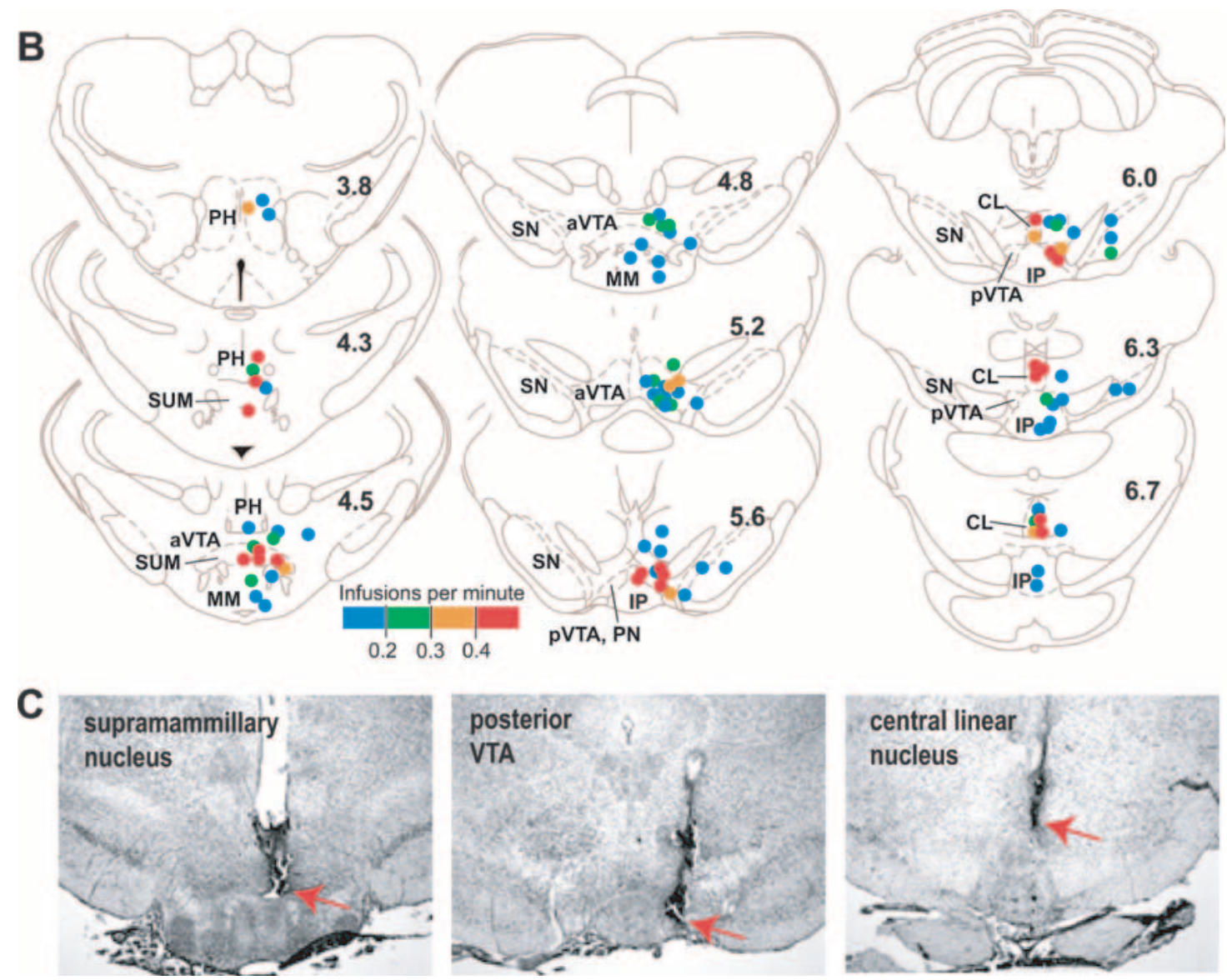

Figure 1. (continued) Regional differences in nicotine self-administration. $A$, Self-administration rates (mean infusions per minute with SEM) are depicted as a function of region and session (concentration). A significant interaction between region and treatment $\left(F_{(7,77)}=3.08 ; p<0.01\right.$ ) was found for the first four sessions. ${ }^{\#} p<0.05$, significantly different compared with respective vehicle trials. A two-way ANOVA with region and high concentration set $(12.5,25$, and 50 or sessions $5-7)$ found that a significant region-by-concentration interaction $\left(F_{(14,154)}=2.90 ; p<0.001\right)$. $\& \& p<0.05$, significantly different compared with anterior VTA, medial mammillary nucleus, region dorsal to posterior VTA-lateral to central linear nucleus (CL), and substantia nigra groups. Similarly, a two-way ANOVA with region and low concentration set $(0.1,0.3,1,3$, and 10 or sessions $9-13)$ found a significant region-by-concentration interaction $\left(F_{(28,308)}=2.63 ; p<0.0001\right)$. ${ }^{* *} p<0.05$, significantly different compared with anterior VTA, medial mammillary nucleus, region dorsal to posterior VTA-lateral to central linear nucleus, and substantia nigra groups. ${ }^{*} p<0.05$, significantly different compared with the medial mammillary nucleus and substantia nigra. $\boldsymbol{B}$, Effectiveness of each injection site is depicted on coronal drawings [adapted and modified from the atlas by Paxinos and Watson (1997)]. The location of each injection cannula tip is shown in a distinct color, which indicates one of four levels of mean self-administration rates during sessions $5-7$. The numbers in the drawings indicate distances from bregma. aVTA, Anterior VTA; CL, central linear nucleus raphé; IP, interpeduncular nucleus; MM, medial mammillary nucleus; PH, posterior hypothalamic nucleus; PN, paranigral nucleus; pVTA, posterior VTA; SN, substantia nigra; SUM, supramammillary nucleus. C, Photomicrograms show the tips of injection cannulas (red arrows) placed for the supramammillary nucleus, posterior VTA, and central linear nucleus.

supramammillary nucleus, although supramammillary region may be less sensitive to nicotine reward than the two midbrain regions. Nicotine was not reinforcing when it was injected into surrounding regions including the region just dorsal to the posterior VTA and just lateral to the central linear nucleus, anterior VTA, substantia nigra, interpeduncular nucleus, or medial mammillary nucleus. The findings that rats discriminated between the active and inactive levers for nicotine infusions into these three regions support the role of nicotine in positive reinforcement. The reinforcing effects of nicotine administration into these sites were mediated via nicotinic receptors, because the blockade of nicotinic receptors attenuated nicotine self-administration.

\section{Methodological issues}

The rates of nicotine self-administration differed when rats received the same concentration of nicotine between the one-lever and two-lever procedures. We would like to address what caused rate differences and how they influence our interpretations of the data. Differences in timeout between the procedures appear to be the major responsible factor. We knew that two-lever procedures with levers available throughout testing would result in high lev- els of inactive lever pressing, and inactive lever presses would increase as a function of active lever presses (Ikemoto et al., 1997a,b, 1998a,b). Thus, such procedures appear to detect drugs' arousing effects reflected in lever pressing. For this reason, both levers were retracted for $20 \mathrm{~s}$ after either active or inactive lever pressing in the present study. The timeout of the two-lever procedure was not only twice as long as that of the one-lever procedure, but an additional timeout was imposed when rats responded on inactive levers. This fact, together with the nicotine concentration, which was still on an ascending limb of the response-concentration curve, appears to have contributed to reducing response levels by shifting the response-concentration curve to the right.

Alternatively, lever presses in the one-lever procedure may have indicated arousal effects that made response levels higher than those present in the two-lever procedure at the same concentration of nicotine. We think that arousal is an essential component of primary reinforcement. Most of, if not all, the drugs known to be intracranially self-administered into the brain appear to induce arousal, as indicated by heightened locomotion or inactive lever pressing. These drugs include opioids or cholin- 
ergic drugs in the posterior VTA (Ikemoto and Wise, 2002; Zangen et al., 2002), muscimol in the central linear nucleus (Ikemoto et al., 1998b), AMPA or GABA receptor antagonists in the supramammillary nucleus (Ikemoto et al., 2004; Ikemoto, 2005a), and dopaminergic or cholinergic drugs in the ventral striatum (Austin and Kalivas, 1988; Ikemoto et al., 1998a, 2005; Ikemoto, 2002, 2003). We suspect that when arousing effects are eliminated from the procedure by an extended timeout, rats will stop self-administration; we did not explicitly design our procedure to eliminate arousal effects on lever pressing. In any case, the differences in response rates between the two procedures should not impose any added difficulty on interpretation of the effects of anti-cholinergic or dopaminergic manipulations.

\section{Circuitry of nicotine reward}

The reinforcing effects of nicotine administered into the posterior VTA or central linear nucleus appear to be mediated via the activation of the mesolimbic dopamine system, which has been implicated in primary reinforcement of psychomotor stimulants. The central linear nucleus raphé [or caudal linear nucleus of the raphé according to Paxinos and Watson (1997)] lies in the midline, at the posterior end of the A10 dopamine nucleus, and contains dopaminergic neurons projecting to the nucleus accumbens (Swanson, 1982). This region is not always distinguished from the VTA (Oades and Halliday, 1987) or even recognized (Pellegrino and Cushman, 1967) and has been ignored from functional investigations. We adapted the definition of Swanson (1982) and distinguished this region from the VTA in the hope of improving our understanding of reinforcement mechanisms. Our present findings as well as the previous finding that the stimulation of $\mathrm{GABA}_{\mathrm{A}}$ receptors in this region is reinforcing (Ikemoto et al., 1998b) suggest that this structure is involved in positive reinforcement.

Most of the findings of the previous studies as well as our present results are consistent with the idea that the rewarding effects of nicotine are mediated by activation of the dopaminergic neurons projecting to the ventral striatum. Nicotine administration into the VTA has been shown to increase extracellular dopamine levels in the nucleus accumbens (Imperato et al., 1986; Ferrari et al., 2002). Intravenous self-administration of nicotine in rats is attenuated by selective lesions of the dopamine neurons projecting to the ventral striatum (Corrigall et al., 1992). Local application of dopaminergic drugs into the nucleus accumbens or olfactory tubercle is sufficient for positive reinforcement (Ikemoto and Wise, 2004). In the present study, we found that the reinforcing effects of nicotine administration into the posterior VTA or the central linear nucleus were attenuated by coadministration of the $\mathrm{D}_{2}$ receptor agonist quinpirole, which inhibits dopaminergic neurons via $\mathrm{D}_{2}$ autoreceptors, as well as by systemic

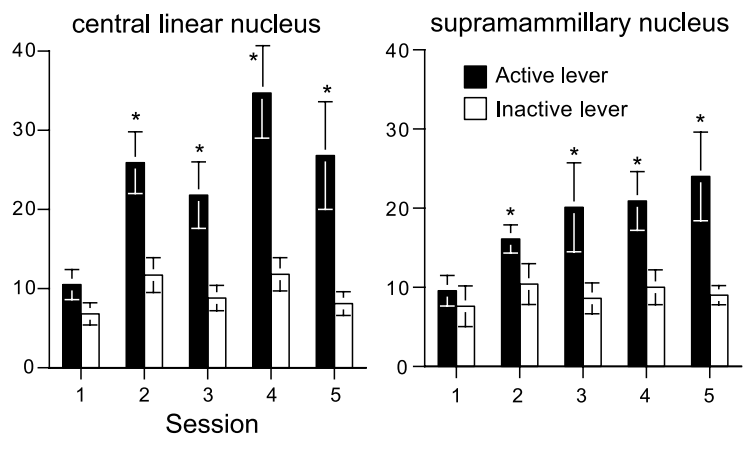

Figure 2. Two-lever discrimination. Data are means plus SEM. Rats received vehicle in session 1 and 25 mm nicotine in sessions 2-5. ANOVAs with two levers over five sessions found that significant lever-by-session interactions for the VTA $\left(n=13 ; F_{(4,48)}=\right.$ , central linear nucleus $\left(n=13 ; F_{(4,48)}=2.76 ; p<0.05\right)$, and supramammillary nucleus $\left(n=9 ; F_{(4,32)}=2.99 ;\right.$ $p<0.05)$. Dunnett's post hoc tests found that active lever presses in session $2-5$ were significantly greater than control lever presses in session $1\left(^{*} p<0.05\right)$, which did not differ from active lever presses in session 1 or control lever presses in sessions $2-5$.

Figure 3. Effects of cholinergic antagonists on nicotine self-administration. Data are means plus SEM. Rats $(n=7)$ self$\left(F_{(2,12)}=5.81 ; p<0.05\right)$ and supramammillary nucleus $\left(F_{(2,14)}=8.43 ; p<0.01\right) .{ }^{*} p<0.05$, significant difference compared with nicotine alone. n., Nucleus; VEH, vehicle.

administration of the $\mathrm{D}_{1}$ receptor antagonist $\mathrm{SCH}$ 23390. Our present findings and many previous findings, however, are not consistent with the recent report that systemic or intraaccumbens administration of dopamine receptor antagonists enhanced the rewarding effects of nicotine administered into the VTA, as indicated by conditioned place preference (Laviolette and van der Kooy, 2003). A number of conditions differ between the present study and the Laviolette and van der Kooy study, which make it difficult to pinpoint the causes of this discrepancy. Additional studies are needed to examine under what conditions the blockade of dopamine transmission enhances the rewarding effects of nicotine.

The anterior VTA and substantia nigra, which contain dopaminergic neurons projecting to the striatum, do not appear to be involved in triggering the reinforcing effects of nicotine. These findings are consistent with previous findings that these regions, unlike the posterior VTA, do not readily respond to the rewarding effects of carbachol, endomorphin-1, ethanol, or acetaldehyde (Rodd-Henricks et al., 2000; Ikemoto and Wise, 2002; Zangen et al., 2002; Rodd et al., 2004). The differences of these regions in sensitivity to drug reinforcement may be explained by differences in the efferent projections to the striatum of these regions. We recently found that the posterior VTA predomi- 
A

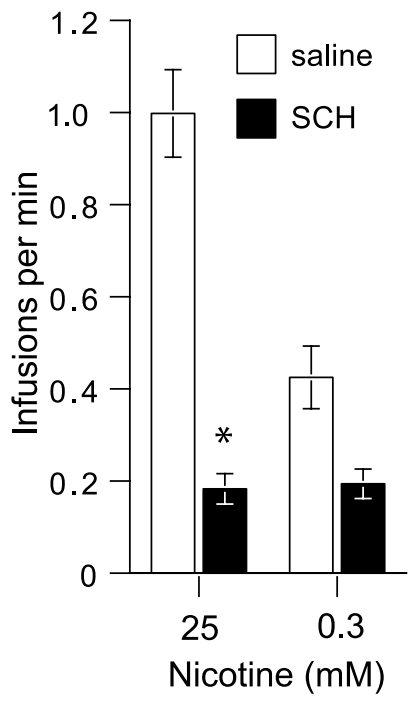

B

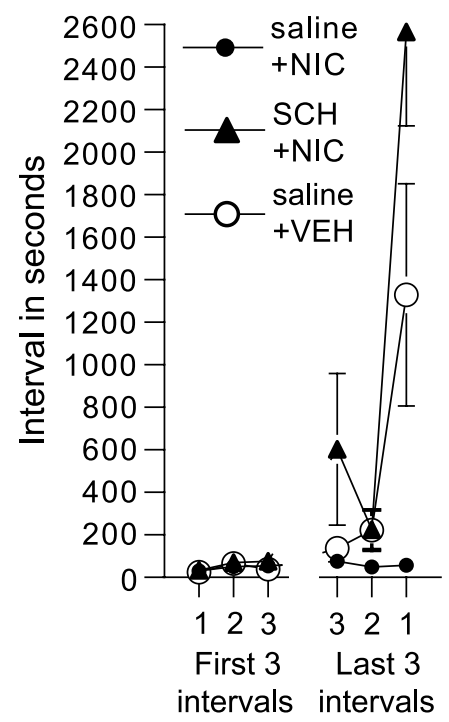

C

saline + VEH alone

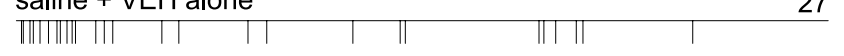

saline $+25 \mathrm{mM}$ nicotine

60

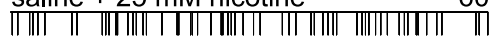

$\mathrm{SCH}+25 \mathrm{mM}$ nicotine

$|\|||||||||\|||||||| \mid$ |

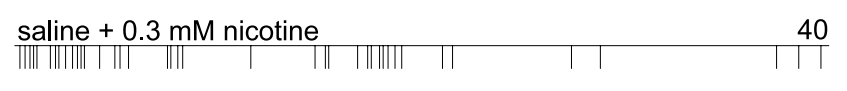

$\mathrm{SCH}+0.3 \mathrm{mM}$ nicotine 21

\section{\|\|\|\||||||| \\ $30 \mathrm{~min}$}

D
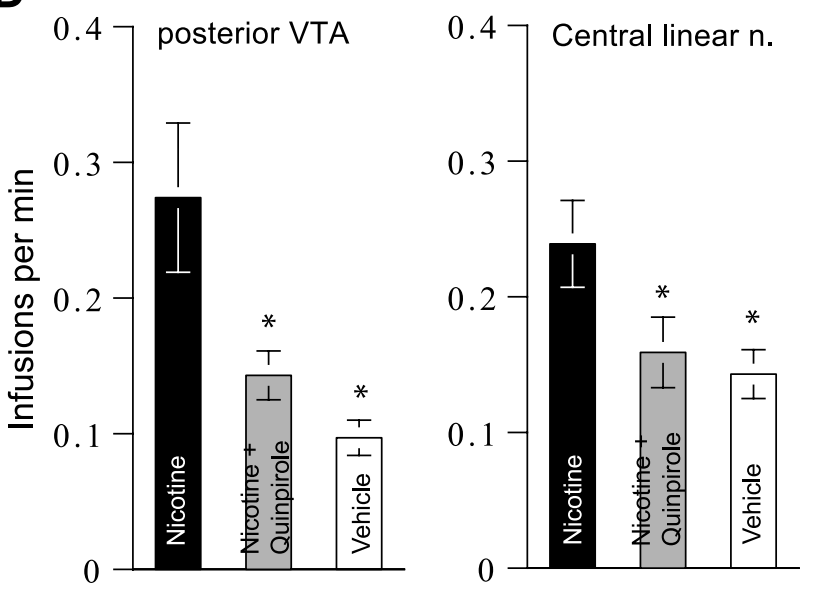

Figure 4. Role of dopamine in nicotine self-administration into the ventromedial midbrain. Rats self-administered 25 or $0.3 \mathrm{~mm}$ nicotine after treated with the $\mathrm{D}_{1}$ antagonist SCH 23390 $(\mathrm{SCH})(0.05 \mathrm{mg} / \mathrm{kg}$, i.p.) or saline $(1 \mathrm{ml} / \mathrm{kg}) . \boldsymbol{A}$, Mean infusion rates with SEM $(n=7)$ are shown. The SCH 23390 treatment significantly reduced the rate of self-infusion of the high nicotine concentration into the posterior VTA $\left({ }^{*} p<0.001\right.$, after a significant interaction between concentration and treatment; $\left.F_{(1,6)}=17.42 ; p<0.01\right)$. In addition, the treatment did not enhance nicotine self-administration but tended to reduce self-administration rates of the low nicotine concentration ( $p=0.058$ ). $\boldsymbol{B}$, Mean infusion intervals with SEM as a function of treatment are nantly projects to the medial part of the nucleus accumbens shell and medial olfactory tubercle (Ikemoto, 2005b), at which dopaminergic drugs such as amphetamine or cocaine readily trigger reinforcing effects (Ikemoto, 2003; Ikemoto et al., 2005). In addition, we found that the central linear nucleus, like the posterior VTA, projects to the medial shell and medial tubercle. On the other hand, the anterior VTA and substantia nigra pars compacta provide little or no projections to these, but instead project to the accumbens core, ventral shell, lateral tubercle, and dorsal striatum, into which the application of dopaminergic drugs is not reinforcing.

The present study also suggests that the supramammillary nucleus of the posterior hypothalamus mediates the reinforcing effects of nicotine. Recent studies found that this structure mediates the reinforcing effects of $\mathrm{GABA}_{\mathrm{A}}$ receptor antagonists and the excitatory amino acid AMPA (Ikemoto et al., 2004; Ikemoto, 2005a), suggesting that supramammillary neurons are important relays of reward circuitry. There is a similarity in afferents between the supramammillary nucleus and VTA. The supramammillary nucleus appears to receive cholinergic inputs from the laterodorsal tegmental nucleus and the medial septal/diagonal band complex (Gonzalo-Ruiz et al., 1999), whereas the VTA (probably the central linear nucleus as well) receives inputs from the laterodorsal and pedunculo pontine tegmental nuclei (Oakman et al., 1995; Omelchenko and Sesack, 2005). On the other hand, efferent projections markedly differ between the regions. The supramammillary nucleus does not project to the nucleus accumbens or olfactory tubercle, but to the septal and hippocampal complexes (Vertes, 1992), which have not been implicated clearly in primary reinforcement. Therefore, nicotine administration into the supramammillary nucleus does not appear to stimulate the known dopamine reward system or at least does not do so directly. Future investigations should explore which neural pathways are involved in positive reinforcement mediated via the supramammillary nucleus.

\section{Implication and conclusion}

The three brain regions that we identified for nicotine reinforcement may play an important role in tobacco-smoking habits. Although the present study found that the stimulation of nicotinic receptors in the posterior VTA, central linear nucleus, or supramammillary nucleus is sufficient to trigger positive reinforcement, it is not clear whether these regions are necessary for reinforcement of tobacco smoking. This issue should be addressed by future research.

We found that the reinforcing effects of nicotine can be trig-

\footnotetext{
$\leftarrow$

shown. The first three infusion intervals did not differ under three treatment conditions: nicotine infusion (NIC) with the $D_{1}$ antagonist pretreatment, nicotine infusion with saline pretreatment, or vehicle infusion (VEH) with saline pretreatment, whereas the last three intervals differed significantly among the treatments (a significant interaction between treatment and first-last interval; $\left.F_{(2,12)}=14.08 ; p<0.001\right)$. When rats received nicotine with $\mathrm{SCH} 23390$ treatment or vehicle with saline treatment, they self-administered infusions at significantly longer intervals than when they received nicotine with saline treatment during the last three intervals $(p<0.01)$. C, Event records of a representative rat are shown. Each vertical line on the horizontal line indicates the time of an infusion. The number just right of the horizontal line indicates total infusions per session. VEH, Vehicle; $\mathrm{SCH}, \mathrm{SCH} 23390$. D, Coadministration of the $\mathrm{D}_{2}$ receptor agonist quinpirole into the posterior VTA $(n=13)$ or central linear nucleus $(\mathrm{n}$.) $(n=$ 6), which inactivates local dopamine neurons, reduced nicotine self-administration. Repeated ANOVAs with nicotine, nicotine plus quinpirole, and vehicle found significant main effects for the VTA $\left(F_{(2,24)}=8.79 ; p<0.05\right)$ and central linear nucleus $\left(F_{(2,10)}=4.59 ; p<0.05\right) .{ }^{*} p<$ 0.05 , significant difference compared with nicotine alone.
} 
gered via multiple regions in the ventral midbrain and the posterior hypothalamus. The study provides clear evidence for anatomical complexity of reinforcement mechanisms in the vicinity of the VTA.

\section{References}

Austin MC, Kalivas PW (1988) The effect of cholinergic stimulation in the nucleus accumbens on locomotor behavior. Brain Res 441:209-214.

Bolanos CA, Perrotti LI, Edwards S, Eisch AJ, Barrot M, Olson VG, Russell DS, Neve RL, Nestler EJ (2003) Phospholipase C $\gamma$ in distinct regions of the ventral tegmental area differentially modulates mood-related behaviors. J Neurosci 23:7569-7576.

Carlezon Jr WA, Haile CN, Coppersmith R, Hayashi Y, Malinow R, Neve RL, Nestler EJ (2000) Distinct sites of opiate reward and aversion within the midbrain identified using a herpes simplex virus vector expressing GluR1. J Neurosci 20:RC62(1-5).

Corrigall WA, Franklin KB, Coen KM, Clarke PB (1992) The mesolimbic dopaminergic system is implicated in the reinforcing effects of nicotine. Psychopharmacology (Berl) 107:285-289.

Corrigall WA, Coen KM, Adamson KL (1994) Self-administered nicotine activates the mesolimbic dopamine system through the ventral tegmental area. Brain Res 653:278-284.

Di Chiara G (2000) Role of dopamine in the behavioural actions of nicotine related to addiction. Eur J Pharmacol 393:295-314.

Ferrari R, Le Novere N, Picciotto MR, Changeux JP, Zoli M (2002) Acute and long-term changes in the mesolimbic dopamine pathway after systemic or local single nicotine injections. Eur J Neurosci 15:1810-1818.

Gonzalo-Ruiz A, Morte L, Flecha JM, Sanz JM (1999) Neurotransmitter characteristics of neurons projecting to the supramammillary nucleus of the rat. Anat Embryol (Berl) 200:377-392.

Ikemoto S (2002) Ventral striatal anatomy of locomotor activity induced by cocaine, D-amphetamine, dopamine and D1/D2 agonists. Neuroscience 113:939-955.

Ikemoto S (2003) Involvement of the olfactory tubercle in cocaine reward: intracranial self-administration studies. J Neurosci 23:9305-9311.

Ikemoto S (2005a) The supramammillary nucleus mediates primary reinforcement via $\operatorname{GABA}(\mathrm{A})$ receptors. Neuropsychopharmacology 30:1088-1095.

Ikemoto S (2005b) The organization of midbrain projections to the ventral striatum in the rat: implication for reward circuitry. Soc Neurosci Abstr 31:75.21.

Ikemoto S, Sharpe LG (2001) A head-attachable device for injecting nanoliter volumes of drug solutions into brain sites of freely moving rats. J Neurosci Methods 110:135-140.

Ikemoto S, Wise RA (2002) Rewarding effects of the cholinergic agents carbachol and neostigmine in the posterior ventral tegmental area. J Neurosci 22:9895-9904.

Ikemoto S, Wise RA (2004) Mapping of chemical trigger zones for reward. Neuropharmacology 47:190-201.

Ikemoto S, Murphy JM, McBride WJ (1997a) Self-infusion of GABA antagonists directly into the ventral tegmental area and adjacent regions. Behav Neurosci 111:369-380.

Ikemoto S, Glazier BS, Murphy JM, McBride WJ (1997b) Role of $\mathrm{D}_{1}$ and $\mathrm{D}_{2}$ receptors in the nucleus accumbens in mediating reward. J Neurosci 17:8580-8587.

Ikemoto S, Glazier BS, Murphy JM, McBride WJ (1998a) Rats selfadminister carbachol directly into the nucleus accumbens. Physiol Behav 63:811-814.

Ikemoto S, Murphy JM, McBride WJ (1998b) Regional differences within the rat ventral tegmental area for muscimol self-infusions. Pharmacol Biochem Behav 61:87-92.
Ikemoto S, Witkin BM, Zangen A, Wise RA (2004) Rewarding effects of AMPA administration into the supramammillary or posterior hypothalamic nuclei but not the ventral tegmental area. J Neurosci 24:5758-5765.

Ikemoto S, Qin M, Liu Z-H (2005) The functional divide for primary reinforcement of D-amphetamine lies between the medial and lateral ventral striatum: is the division of the accumbens core, shell and olfactory tubercle valid? J Neurosci 25:5061-5065.

Imperato A, Mulas A, Di Chiara G (1986) Nicotine preferentially stimulates dopamine release in the limbic system of freely moving rats. Eur J Pharmacol 132:337-338.

Laviolette SR, van der Kooy D (2003) Blockade of mesolimbic dopamine transmission dramatically increases sensitivity to the rewarding effects of nicotine in the ventral tegmental area. Mol Psychiatry 8:50-59.

Maskos U, Molles BE, Pons S, Besson M, Guiard BP, Guilloux JP, Evrard A, Cazala P, Cormier A, Mameli-Engvall M, Dufour N, Cloez-Tayarani I, Bemelmans AP, Mallet J, Gardier AM, David V, Faure P, Granon S, Changeux JP (2005) Nicotine reinforcement and cognition restored by targeted expression of nicotinic receptors. Nature 436:103-107.

Museo E, Wise RA (1994) Place preference conditioning with ventral tegmental injections of cytisine. Life Sci 55:1179-1186.

Oades RD, Halliday GM (1987) Ventral tegmental (A10) system: neurobiology. 1. Anatomy and connectivity. Brain Res Brain Res Rev 12:117-165.

Oakman SA, Faris PL, Kerr PE, Cozzari C, Hartman BK (1995) Distribution of pontomesencephalic cholinergic neurons projecting to substantia nigra differs significantly from those projecting to ventral tegmental area. J Neurosci 15:5859-5869.

Olson VG, Zabetian CP, Bolanos CA, Edwards S, Barrot M, Eisch AJ, Hughes T, Self DW, Neve RL, Nestler EJ (2005) Regulation of drug reward by cAMP response element-binding protein: evidence for two functionally distinct subregions of the ventral tegmental area. J Neurosci 25:5553-5562.

Omelchenko N, Sesack SR (2005) Laterodorsal tegmental projections to identified cell populations in the rat ventral tegmental area. J Comp Neurol 483:217-235.

Paxinos G, Watson C (1997) The rat brain in stereotaxic coordinates, Ed 3. San Diego: Academic.

Pellegrino LJ, Cushman AJ (1967) A stereotaxic atlas of the rat brain. New York: Appleton-Century-Crofts.

Phillipson OT (1979) The cytoarchitecture of the interfascicular nucleus and ventral tegmental area of Tsai in the rat. J Comp Neurol 187:85-98.

Picciotto MR, Corrigall WA (2002) Neuronal systems underlying behaviors related to nicotine addiction: neural circuits and molecular genetics. J Neurosci 22:3338-3341.

Rodd-Henricks ZA, McKinzie DL, Crile RS, Murphy JM, McBride WJ (2000) Regional heterogeneity for the intracranial self-administration of ethanol within the ventral tegmental area of female Wistar rats. Psychopharmacology (Berl) 149:217-224.

Rodd ZA, Bell RL, Zhang Y, Murphy JM, Goldstein A, Zaffaroni A, Li TK, McBride WJ (2004) Regional heterogeneity for the intracranial selfadministration of ethanol and acetaldehyde within the ventral tegmental area of alcohol-preferring $(\mathrm{P})$ rats: involvement of dopamine and serotonin. Neuropsychopharmacology 30:330-338.

Swanson LW (1982) The projections of the ventral tegmental area and adjacent regions: a combined fluorescent retrograde tracer and immunofluorescence study in the rat. Brain Res Bull 9:321-353.

Vertes RP (1992) PHA-L analysis of projections from the supramammillary nucleus in the rat. J Comp Neurol 326:595-622.

Zangen A, Ikemoto S, Zadina JE, Wise RA (2002) Rewarding and psychomotor stimulant effects of endomorphin-1: anteroposterior differences within the ventral tegmental area and lack of effect in nucleus accumbens. J Neurosci 22:7225-7233. 